\title{
Experimental micromechanics: grain-scale observation of sand deformation
}

\author{
E. ANDÒ*, S. A. HALL†, G. VIGGIANI*, J. DESRUES* and P. BÉSUELLE*
}

\begin{abstract}
Strain localisation plays a key role in the deformation of granular materials. Such localisation involves bands of just a few grains wide, which dominate the material's macroscopic response. This grainscale phenomenon presents challenges for continuum modelling, which is the rationale behind models that explicitly take micro-scales into account. These in turn require micro-scale experimental analysis. In this work, X-ray tomography is used to image a small sample of oolitic sand while it deforms under triaxial compression. Grains are followed with a technique combining recent developments in image correlation and particle tracking. From these rich data, the evolution of the material in a subvolume of a thousand grains inside the sample (which contains 53000 grains) is presented. The subvolume is chosen to lie inside the shear band that appears at the sample scale. Three-dimensional (3D) grain kinematics are analysed in three increments: the beginning of the test, the peak of the sample's macroscopic axial stress response and the residual stress state. When the sample's deformation is homogeneous (increment one) or fully localised (increment three), the kinematics of the grains in the subvolume appear to be representative of the kinematics occurring at the sample scale, allowing micro-mechanical observations to be made. In the transition from homogeneous to localised deformation (increment two), however, the scale of observation requires a zoom out of the subvolume to the sample scale in order to capture the complex mechanisms at play.
\end{abstract}

KEYWORDS: laboratory tests; sand; strain localisation

ICE Publishing: all rights reserved

\section{INTRODUCTION}

From a mechanical standpoint, granular materials exhibit complex behaviour. One major complexity is the loss of homogeneity of the deformation process during loading. Strain localisation, which precedes and leads to global failure, operates at a micro-scale characteristic of the material. The central role played by grain-scale mechanisms in the onset and evolution of shear bands in granular materials has been a subject of study for some time (e.g. Mühlhaus \& Vardoulakis, 1987; Iwashita \& Oda, 2000; Alonso-Marroquin \& Vardoulakis, 2005; Fu \& Dafalias, 2012).

In modelling the mechanical behaviour of materials, one approach to handle strain localisation is to incorporate a micro-scale. In Grenoble this has been approached in different ways, for example by using higher-order continua (e.g. second gradient models, see Chambon (2011)), or multi-scale computations (in which a micro-scale is explicitly simulated, e.g. Nitka et al. (2011) and Andrade et al. (2011)). In order to inform these models, the mechanisms involved with strain localisation need to be experimentally investigated at the micro-scale. In the case of sand, the characteristic micro-scale of interest is most likely the grain-scale. Historically, behaviour at this scale has been investigated predominantly using simulations of simplified grains (such as the discrete element method

Manuscript received 7 May 2012; first decision 8 June 2012; accepted 18 July 2012

Published online at www.geotechniqueletters.com on 28 September 2012

*Grenoble-INP/UJF-Grenoble 1/CNRS UMR 5521, Laboratoire 3SR, 38041 Grenoble, France

$\dagger$ †ivision of Solid Mechanics, Lund University, Lund, Sweden and European Spallation Source AB, Lund, Sweden
(DEM)), rather than experiments, which as Sibille \& Froiio aptly stated: "has lead to the paradox of micromechanics of granular materials as a science based almost entirely on "virtual evidence" (Sibille \& Froiio, 2007).

Experimental measurement techniques in geomechanics testing have progressed enormously over the last decades (see the review paper by Viggiani \& Hall (2008) and references cited therein for an historical perspective). X-ray tomography now allows the evolution of the threedimensional (3D) microstructure of a small sample of sand to be followed while it deforms. Individual grains can be distinguished in such time-lapse 3D images and the full 3D kinematics of all the grains can now be measured: a new technique, combining digital image correlation (DIC) (inspired from Hall et al. (2010)) and particle tracking (Andò et al., 2012) is used to make these measurements on a triaxial compression test on an oolitic sand. Rather than analysing the whole sample (as done previously), the analysis here focuses on a cubic subvolume of a thousand grains extracted from the centre of the sample (chosen to sit across the shear band that crosses the sample by the end of the test). The selection of a limited amount of grains allows an easier grain-by-grain inspection of results, and is also done in the spirit of facilitating a direct comparison with results from DEM in the future. The kinematics (3D displacements and rotations) of this assembly of grains is analysed. A few selected results are presented, the main objective being the presentation of the method and the grain scale characterisation thus possible.

\section{EXPERIMENTAL METHODS}

The test described in this paper is a standard triaxial compression test except for the very small sample size 
(11 $\mathrm{mm}$ diameter, $24 \mathrm{~mm}$ height). The apparatus used is described in Hall et al. (2010). In essence, the triaxial apparatus (made from poly(methyl methacrylate)), is practically the same as a conventional system, except the much smaller size and the shape of the confining cell. The tensile reaction force is carried by the cell walls rather than by tie bars. The axial load and hence the deviator stress are applied using a motor-driven screw actuator, which does not interfere with the tomographic X-ray scans. The material tested is an oolitic sand from Caicos (British West Indies), a natural material made up of round calcite grains. Samples are prepared by pluviation of the oolite grains into a thin latex membrane. The sample is tested dry at a constant cell pressure of $100 \mathrm{kPa}$. Figure 1 shows the grain size distribution calculated from the equivalent sphere diameter of all the 53000 grains of the sample, as well as a cross-section from a nano-tomography performed on a single grain showing it is rounded.

The sample is sheared under displacement control (axial loading is applied upwards on the bottom of the sample) at $21 \mu \mathrm{m} / \mathrm{min}$. Axial loading is interrupted at several points during the test to perform X-ray scans (at a resolution of $15.6 \mu \mathrm{m} / \mathrm{pixel}$ ), to give 3D images of the sample in each state (plus one at the beginning of the test, before shearing).

\section{MEASURING GRAIN KINEMATICS}

In order to be able to study deformation at the grain-scale, individual grains must be identified in the $3 \mathrm{D}$ images coming from X-ray tomography. To this end images are binarised - assigning each voxel either to pore or to grain, based on their greyscale value. This creates a single interconnected grain mass, which is then split into separate grains using a Watershed algorithm (Marak et al., 2011), starting from markers automatically positioned inside each grain (which works best for objects close to spherical - this explains the choice of a rounded sand). For the purposes of this work, a few of the markers are modified by hand in order to give the best segmentation for this relatively small sub-volume. The lines that separate touching grains are recorded as surfaces of contact. Once grains are split apart, each one is assigned a unique label. Particle tracking (IDTrack, Andò et al., 2012) is then used to track grains, from one configuration (scan) to the next. Grains are identified in a neighbourhood from one configuration to the next by their individual volume. The $3 \mathrm{D}$ displacement of each grain is given by the change in the position of its centre of mass between configurations. It is measured with an expected error of around $1.5 \mu \mathrm{m}$ in these conditions (i.e. $0 \cdot 1$ pixels, see Andò et al., 2012).

The $3 \mathrm{D}$ grain rotations are determined from the change in a grain's orientation between configurations, which was

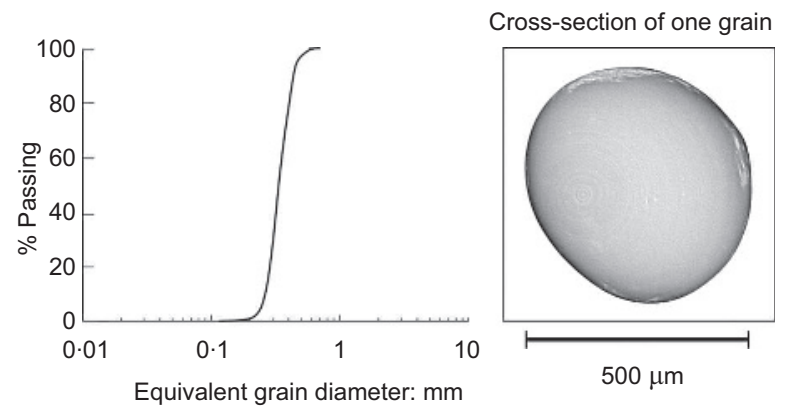

Fig. 1. Left: grain-size distribution of the sample tested. Right: slice of X-ray nano-tomography on a single grain of Caicos ooid, courtesy of RX-Solutions (France) previously described using the major and minor eigenvectors of the grain's moment of inertia tensor, simply calculated on the entirety of voxels describing the grain. Such an approach provides very fast computation (as the change in orientation between configurations is simply calculated by comparing two three-component vectors for each configuration). However, these vectors have been observed to be vulnerable to non-physical changes between configurations. These changes are thought to be either due to the roundness of grains meaning that minimum or maximum directions are poorly defined, or where they are well-defined (in angular grains for example) $180^{\circ}$ shifts can occur in either vector, as there is no pre-defined direction the latter is corrected to some extent by 'folding' rotations around $180^{\circ}$. A new hybrid, grain-based image-correlation approach has therefore been developed, which matches images of the grains between configurations (the images of the grains contain several thousand data points rather than two three-component vectors), in the style of Hall et al. (2010). This approach skips the computationally expensive searching of the grains by image correlation, and instead uses the results of ID-Track to find the images of a grain in reference and deformed configurations, which are then copied into padded cubic arrays. The reference image is rotated and displaced in $3 \mathrm{D}$ by transforming its original coordinates and looking them up in a specifically written trilinear interpolation of the reference image. The search for the three rotation and three displacement components yielding the highest similarity between the rotated and translated reference image and the deformed image (i.e. the highest normalised correlation coefficient (Lewis, 1995) is then led by a BFGS optimisation algorithm (as available, for example, in Nocedal \& Wright (1999)). On an Intel Core $i 7$ the image correlation takes about $10 \mathrm{~s}$ per grain, generating around 200 interpolated images before finding a minimum. The performance of this measurement technique can be seen in Fig. 2, which shows the measurements for approximately 5000 grains subjected to a $4 \cdot 7^{\circ}$ rigid body rotation. The improvement in the measurement compared to the previous technique is obvious, the histogram is now spread over only a few degrees.

\section{SELECTED RESULTS}

The global sample stress-strain response is presented in Fig. 3 (top). The sample responds as expected for a dense sand - with a distinct peak in the axial stress response, giving perhaps a surprisingly high friction angle of $47.7^{\circ}$, especially for a rounded material. A possible explanation may be found in the particular nature of these grains; Fig. 1 (right) shows an increased porosity on the outside of the

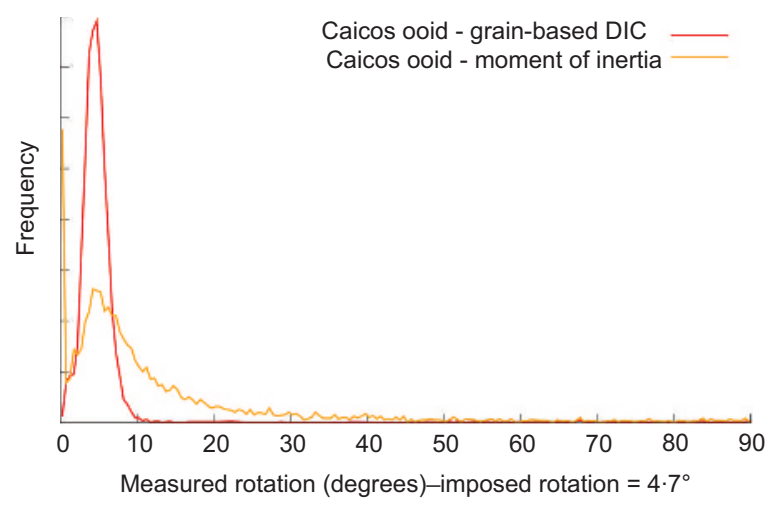

Fig. 2. Histogram of measured rotations with two different techniques for an imposed rigid-body rotation of $4.7^{\circ}$ 
grain, which might crush under load giving rise to a higherthan-expected inter-particle friction. The volumetric strain (calculated from the scans) shows a very small amount of contraction, followed by dilation. There are 16 axial stress relaxations at different values of shortening; these are the points at which the loading has been stopped in order to perform an X-ray scan.

Figure 3 (top) also shows the increments under study:

(1) a small increment (in terms of axial shortening) at the beginning of the test

(2) an increment over the peak in the axial stress response close to the maximum angle of dilatancy

(3) an increment in the residual stress state with little or no stress and volume change.

Figure 3 (bottom) shows the positions of the grains in the subvolume at the beginning of the first increment, and their positions at the end of the last increment. The vertical slice is oriented to contain the axis of the sample and the normal to the single shear band that eventually develops.

Figure 4 (top and middle) shows the evolution of the subvolume, colouring each of the thousand grains by measurements of their vertical displacement and by their angle of rotation (in the axis and angle representation of $3 \mathrm{D}$ rotations) over the three strain increments shown in Fig. 3. The loading piston moves upwards and there is zero-displacement imposed at the top of the sample; negative displacements are upwards, and 1 pixel equals $15.6 \mu \mathrm{m}$. At the beginning of the test (increment 1), the grains in the subvolume show vertical displacements that have a smooth vertical variation, and the measured rotations are very small and not organised in space. In the increment 2 the overall grain displacements show a rotation away from the vertical owing to the shear band that appears macroscopically. Rotations are considerably larger than in the previous increment, but still do not show any particular spatial organisation. The final increment clearly shows the shear band, both in the displacements as well as in the rotations, which now show strong organisation in space - with the concentration of the largest rotations in the middle of the band.

Figure 4 (bottom) shows the magnitude of rotations for the whole sample over the same three increments - using the same slice orientation as in Fig. 3 (bottom). Increments 1 and 3 confirm what can be seen in the rotation of the grains in the sub-volume - no spatial organisation at the beginning, and a concentrated band of rotations at the end. Increment 2 , however, shows that spatial organisation is definitely visible in the rotations at the sample scale. Rotations are concentrated in a region about fifteen grains thick around the region where the shear band will be at the end of the test. Alignment of large rotations can be seen in the direction of the shear band, as well as orthogonal to it. This macroscopic spatial organisation is not visible at the subvolume scale.

\section{CONCLUSIONS}

In this paper, a hybrid measurement technique has been developed for the analysis of 3D images of a deforming granular medium. This has been achieved by adding

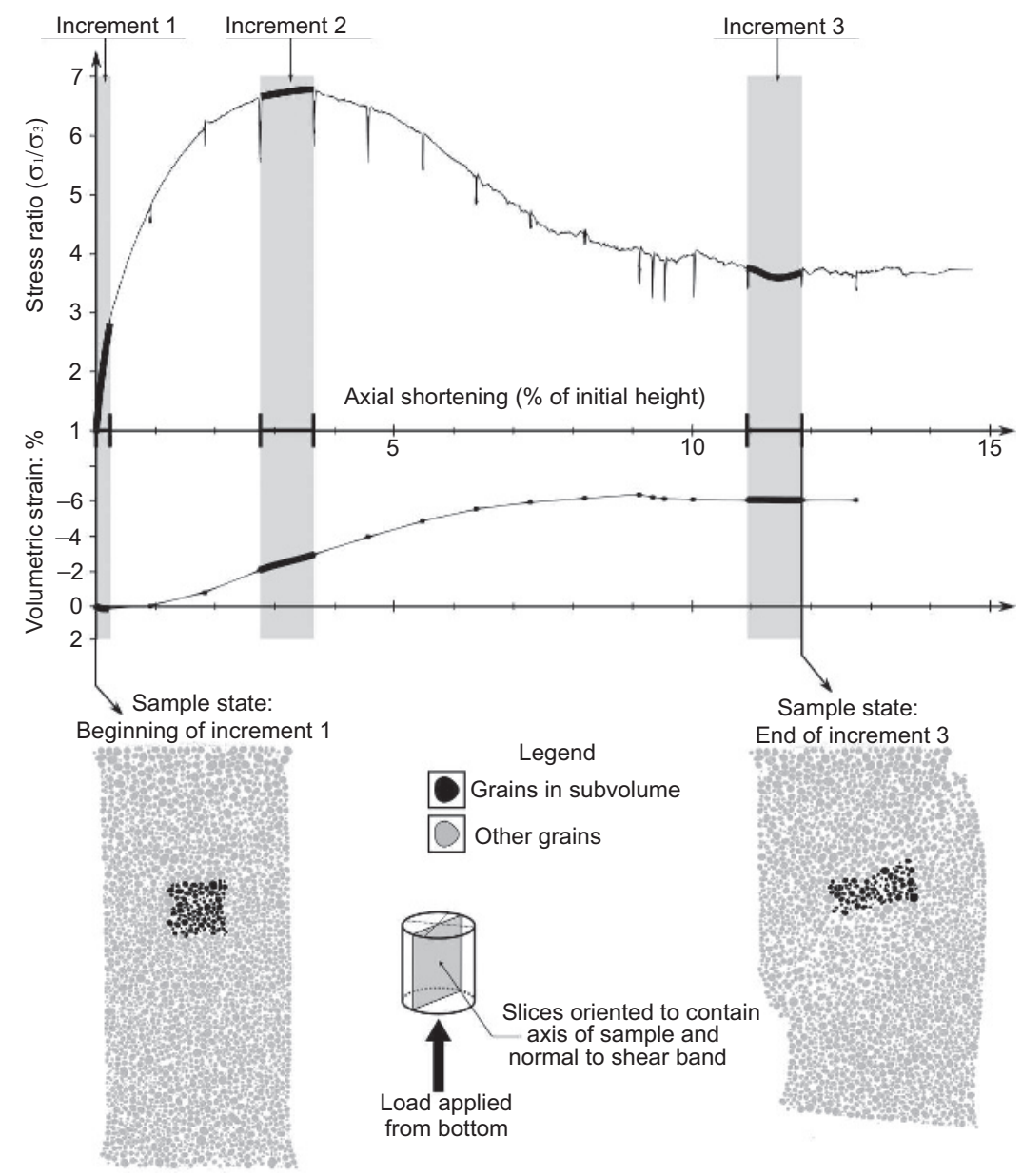

Fig. 3. Top: global axial stress and volumetric strain of the sample against axial shortening. Bottom: vertical slices of $X$-ray scans at beginning and end of the test, showing the location of the grains chosen for this study 

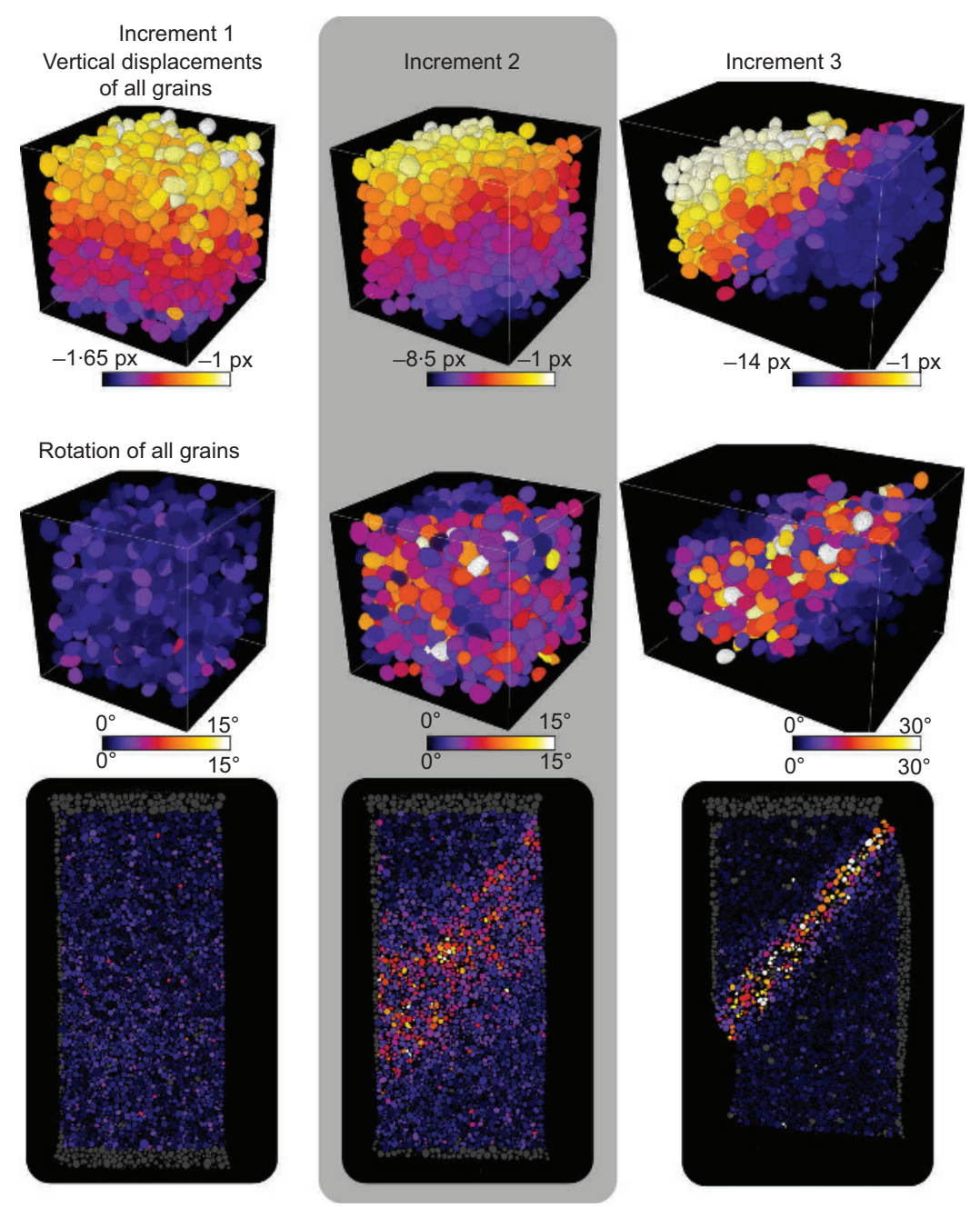

Fig. 4. Top and middle: $3 D$ renderings of the subvolume under study colouring grains. Negative values of vertical displacement are upwards. Bottom: rotations for whole sample shown on a vertical slice (same orientation as in Fig. 3 bottom). Grey grains are not tracked (at the limits of the sample they fall outside the chosen zone of analysis)

grain-based image-correlation for the measurement of rotations, to the previously developed particle tracking technique, ID-Track. This new tool allows the accurate (and relatively rapid) measurement of $3 \mathrm{D}$ grain kinematics. In this paper, it has been applied to images of an oolitic sand sample undergoing triaxial compression, to a small subvolume of a thousand grains as well as the whole sample.

Comparing the kinematics of the subvolume to the overall kinematics in the sample, there is a good correspondence between the two in the increments at the beginning and at the end of the test. At the beginning of the test the sample undergoes homogeneous deformation so the subvolume's kinematics are necessarily representative of those of the sample. At the end of the test a fully developed shear band crosses both the sample and the subvolume, with no deformation outside the band: the subvolume is thus able to capture the kinematics both inside and outside the band, and is therefore representative in some way of the overall kinematics occurring in the sample. However, in the transition from homogeneous to localised deformation (in the increment over the peak in the axial stress response) there is a poor correspondence between the subvolume's kinematics and those seen at the sample scale. Little spatial organisation is visible in the rotations of the grains in the subvolume. At the samplescale, however, some widely distributed, complex patterns of grain rotations are visible around the region where the shear band is at the end of the test. This means that in the transition between these two modes of deformation, the relevant scale of observation of grain kinematics becomes the sample scale. Grain rotations appear to be key for detecting the onset of localisation - which is why this new hybrid measurement technique approach is important.

These experimental results underline the fact that it is risky to study localisation in granular materials with a small numbers of grains: even if localisation occurs, some important phenomena during the change of deformation mode may be missed. Although sample-scale complexity is visible in the experiments presented, it is worth recalling the milestone study by Desrues et al. (1996) where triaxial sand samples of standard size were scanned post-mortem in a medical X-ray tomograph unable to resolve individual grains. Local porosity changes were tracked, revealing complex patterns of localised porosity changes very unlikely to occur with the sample sizes studied in the work presented in this paper. This serves as another reminder that although localised deformation in granular materials is a grain-scale process (and must ideally be characterised as such, with the kind of tools presented herein), it has complex macro-scale manifestations.

A long-term objective is now to study the evolution of the contacts and their role in the mechanics observed, in order to reappraise in $3 \mathrm{D}$ the experimental observations in 2D by Calvetti et al. (1997). This requires accurate 

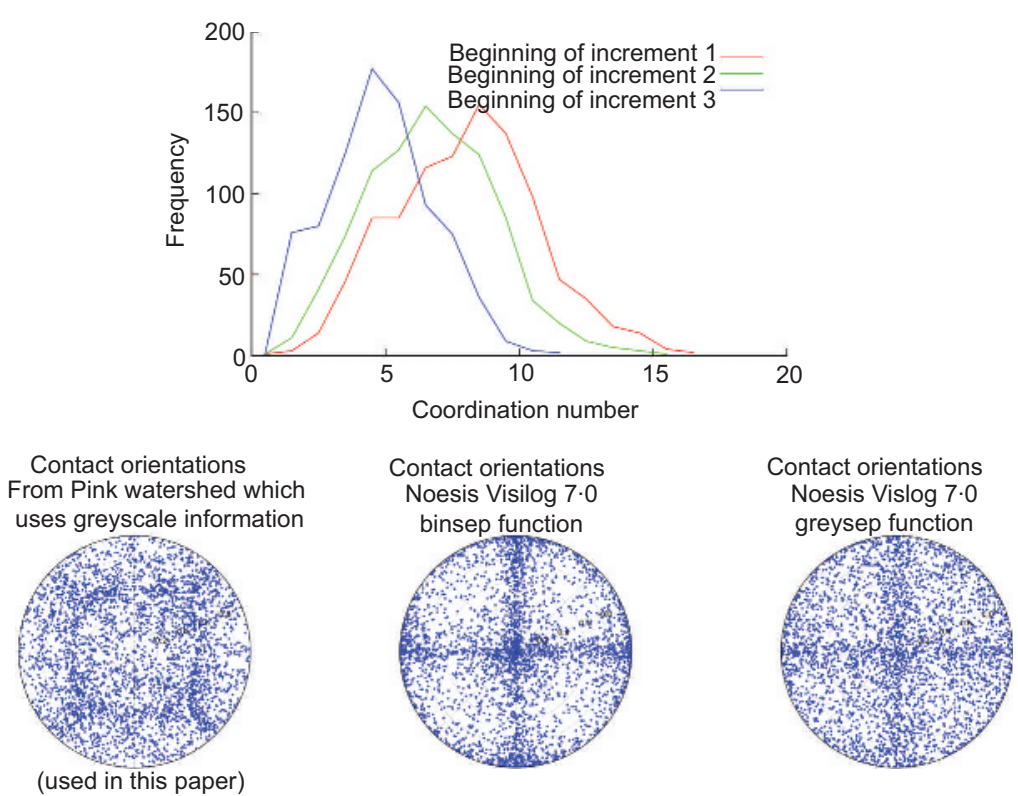

Fig. 5. Top: histogram of coordination number for grains in the subvolume at the beginning of the three increments presented. Bottom: stereonet representations of the orientations for all contacts (approximately 4000) coming from different watershed techniques applied to the same image. Note: these show 3D orientations (a vertical contact is a point in the middle of the circle). An equal-area (Lambert) projection has been used

measurement of (a) presence of contacts; (b) contact orientation; (c) contact kinematics (i.e. sliding, rolling etc.).

In this work, grain contacts are directly given by the watershed segmentation, and grain-to-grain contacts can be counted to determine the number of other grains that each grain is in contact with; that is, its coordination number. Figure 5 (top) shows the evolution of the average coordination number, for grains in the subvolume, in the three increments under study. The average contact number can be seen to consistently decrease during the test, with a drastic overall reduction (approximately half) by the end of the test. The presence of contacts can therefore be measured successfully.

The orientation of a contact, however, is difficult to measure from these images because the spatial resolution $(15.6 \mu \mathrm{m} /$ pixel $)$ of the scans is not enough accurately to resolve the surfaces of the grains in contact. Measured contact orientations are therefore highly dependent on the type of watershed method used to split the grains apart (creating the contact areas by removing voxels at the interface between grains); this is illustrated in Fig. 5 (bottom), where the measured 3D orientations of the contacts in the beginning of the first increment are shown, using different watershed implementations - showing a very strong dependence on the implementation used. As the measurement of grain orientations requires access to the morphology of the grain, it appears that higher resolution data are required to measure contact evolution. More advanced segmentation techniques, as well as the calculation of contact orientations based on grain topology around the contact are currently being investigated, in order to allow the progression into the measurement of contact kinematics. It is likely that the performance of these different techniques will be tested on synthetic images where the contact orientations are known. A correct assessment of contact orientations will then lead to the ability to assess the contact kinematics in the local graincontact reference frame. This will eventually allow a complete characterisation of the kinematics of sand deformation.

\section{REFERENCES}

Alonso-Marroquín, F. \& Vardoulakis, I. (2005). Micromechanics of shear bands in granular media. In Powders and grains (GarcíaRojo R, Herrmann H \& McNamara S (eds)). Leiden, The Netherlands: A.A. Balkema, pp. 701-704.

Andò, E., Hall, S. A., Viggiani, G., Desrues, J. \& Bésuelle, P. (2012). Grain-scale experimental investigation of localised deformation in sand: a discrete particle tracking approach. Acta Geotechnica 7, No. 1, 1-13.

Andrade, J. E., Avila, C. F., Hall, S. A., Lenoir, N. \& Viggiani, G. (2011). Multiscale modeling and characterization of granular matter: From grain kinematics to continuum mechanics. $J$. Mech. Phys. Solids 59, No. 2, 237-250.

Calvetti, F., Combe, G. \& Lanier, J. (1997). Experimental micromechanical analysis of a 2D granular material: relation between structure evolution and loading path. Mech. CohesiveFrictional Mater. 2, No. 2, 121-163.

Chambon, R. (2011). Instability and bifurcation for inelastic geomaterials. In Advances in Bifurcation and Degradation in Geomaterials. Proceedings of the 9th International Workshop on Bifurcation and Degradation in Geomaterials (Bonelli S, Dascalu, $C$ \& Nicot F (eds)), Springer Series in Geomechanics and Geoengineering, 1st edn. New York: Springer, XVII. See http:// www.springer.com/materials/mechanics/book/978-94-007-14205.

Desrues, J., Chambon, R., Mokni, M. \& Mazerolle, F. (1996). Void ratio evolution inside shear bands in triaxial sand specimens studied by computed tomography. Géotechnique 46, No. 3, 529546

Fu, P. \& Dafalias, Y. F. (2012). Quantification of large and localized deformation in granular materials. Int. J. Solids Structs 49, No. 13, 1741-1752.

Hall, S. A., Bornert, M., Desrues, J., Pannier, Y., Lenoir, N., Viggiani, G. \& Bésuelle, P. (2010). Discrete and continuum experimental study of localised deformation in Hostun sand under triaxial compression using X-ray $\mu \mathrm{CT}$ and $3 \mathrm{D}$ digital image correlation. Géotechnique 60, No. 5, 315-322.

Iwashita, K. \& Oda, M. (2000). Micro-deformation mechanism of shear banding process based on modified distinct element method. Powder Technol. 109, No. 1-3, 192-205.

Lewis, J. P. (1995). Fast template matching. Vision Interface 120 123.

Marak, L., Couprie, M. \& Talbot, H. (2011). The Pink image processing library. Proceedings of EuroSciPy - European Conference 
on Scientific Python. Ecole Normale Supérieure, Paris, 25-28 August 2011. For talk, see http://www.euroscipy.org/talk/4190 and for code see https://www.pinkhq.com/homepage/.

Mühlhaus, H. B \& Vardoulakis, I. (1987). The thickness of shear bands in granular materials. Géotechnique 37, No. 3, 271283.

Nitka, M., Combe, G., Dascalu, C. \& Desrues, J. (2011). Twoscale modeling of granular materials: a DEM-FEM approach Granular Matter 13, No. 3, 277-281.
Nocedal, J. \& Wright, S. J. (1999) Numerical optimization. Berlin: Springer, p. 198.

Sibille, L. \& Froiio, F. (2007). A numerical photogrammetry technique for measuring microscale kinematics and fabric in schneebeli materials. Granular Matter 9, 183-193.

Viggiani G. \& Hall, S. A. (2008). Full-field measurements, a new tool for laboratory experimental geomechanics. Keynote paper. Proc. 4th Int. Symp. Deformation Characteristics of Geomaterials. IOS Press, pp. 3-264.

\section{WHAT DO YOU THINK?}

To discuss this paper, please email up to 500 words to the editor at journals@ice.org.uk. Your contribution will be forwarded to the author(s) for a reply and, if considered appropriate by the editorial panel, will be published as a discussion. 